\title{
Assistanat au cabinet médical
}

\author{
François Pilet ${ }^{a}$, Bernard Giorgis ${ }^{b}$
}

a Dr méd., Cursus Romand de Médecine de Famille, membre FMH; ${ }^{b}$ Dr méd., Cursus Romand de Médecine de Famille, membre FMH

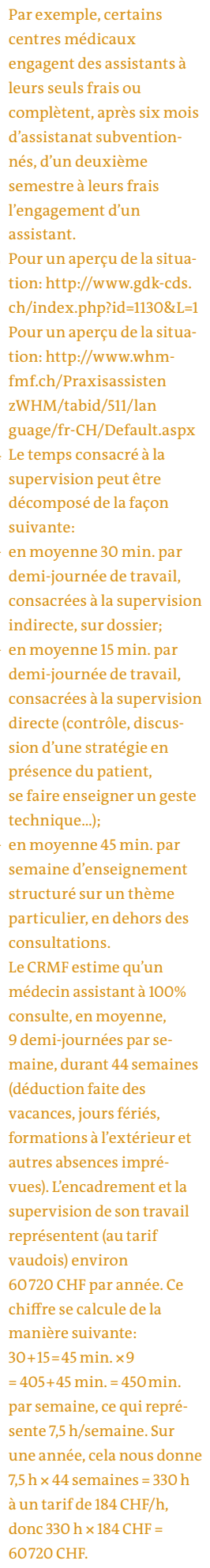

\section{Point de départ}

Depuis une dizaine d'années la corporation médicale et les responsables politiques de santé publique ont pris conscience de la pénurie existante et à venir de médecins de premier recours, en particulier dans les régions périphériques. Ainsi, dans le but de promouvoir la relève auprès des médecins de famille, avec l'appui de la Conférence latine des Affaires Sanitaires et Sociales (CLASS), des programmes correspondants ont été créés dans tous les cantons romands et cofinancés par ceux-ci. Dès 2007, la CLASS a par ailleurs confié au Cursus Romand de Médecine de Famille (CRMF) la responsabilité de développer et coordonner la formation postgraduée en médecine de famille et l'assistanat au cabinet médical en Romandie.

L'idée du programme d'assistanat au cabinet a été lancée en 1998 déjà par la FMH et le Collège de médecine de premier recours (CMPR). En 2008, la responsabilité du programme CMPR a été transmise à la Fondation pour la Promotion de la Formation en Médecine de Famille (FMF) nouvellement créée. En plus des postes d'assistanat au cabinet que cofinancent les cantons, une cinquantaine d'assistanats au cabinet sont cofinancés chaque année dans toute la Suisse par cette fondation.

La création de ces postes répond au constat selon lequel la formation actuelle est essentiellement hospitalo-centrée. La formation spécifique à la médecine ambulatoire est différente de l'acquisition des compétences relevant de l'hôpital. L'assistanat au cabinet est l'un des outils essentiels pour une formation à une médecine ambulatoire efficiente et de qualité.

\section{Aperçu de la situation}

Actuellement, les postes de formation en cabinet médical sont principalement subventionnés et la pratique montre que divers financements existent en Suisse. Deux sources principales de financement coexistent ${ }^{1}$ :

- Les programmes d'assistanat en cabinet médical développés par chaque canton ${ }^{2}$ : les autorités cantonales allouent un financement pour une période de six mois à plein temps ou une année à mi-temps et celui ne peut être attribué qu'une fois par médecin durant l'entier de sa formation. Cette durée a pour logique d'assurer un minimum pour tous dans un contexte d'enveloppe budgétaire limitée.

- Le financement de certains postes par la $\mathrm{FMF}^{3}$ qui répartit un financement provenant de la FMH.

Pour les stages en cabinet médical, les cantons subventionnent actuellement une part de 60 à $80 \%$ du salaire du médecin assistant, en tenant compte des années de pratique professionnelle, sur une base salariale annuelle moyenne de 100000 CHF à plein temps, charges sociales comprises. Cette façon de faire implique donc le financement du temps d'apprentissage.

\section{Proposition du CRMF}

Le financement des heures d'apprentissage ne correspond pas à la situation réelle. En effet, même s'il est en formation, le médecin assistant fournit un travail clinique facturable au patient. En revanche, le maître de stage, pendant son travail de supervision et d'enseignement, ne produit aucun chiffre d'affaire. D'autre part, la pratique en cabinet évolue vers un développement accru de cabinets de groupe et de centres médicaux de plus grande envergure. Ces établissements permettent une professionnalisation de la formation ambulatoire des médecins.

\section{Le financement des heures d'apprentissage ne correspond pas à la situation réelle.}

Face à cette situation, le CRMF propose de renoncer à la notion de subventionnement du salaire de l'assistant et de le remplacer par le financement du travail du maître de stage pour la supervision, l'enseignement et l'encadrement de l'assistant. Ainsi, le CRMF estime qu'un financement forfaitaire de 60000 francs par année ${ }^{4}$ de formation d'un médecin assistant à $100 \%$ paraît équitable, surtout si l'on favorise le développement de la formation des médecins de famille dans des cabinets de groupe. Ce montant correspond à la rétribution du maître de stage pour le temps consacré au médecin en formation. Le salaire du médecin assistant serait, lui, considéré autofinancé par le travail clinique fourni. 


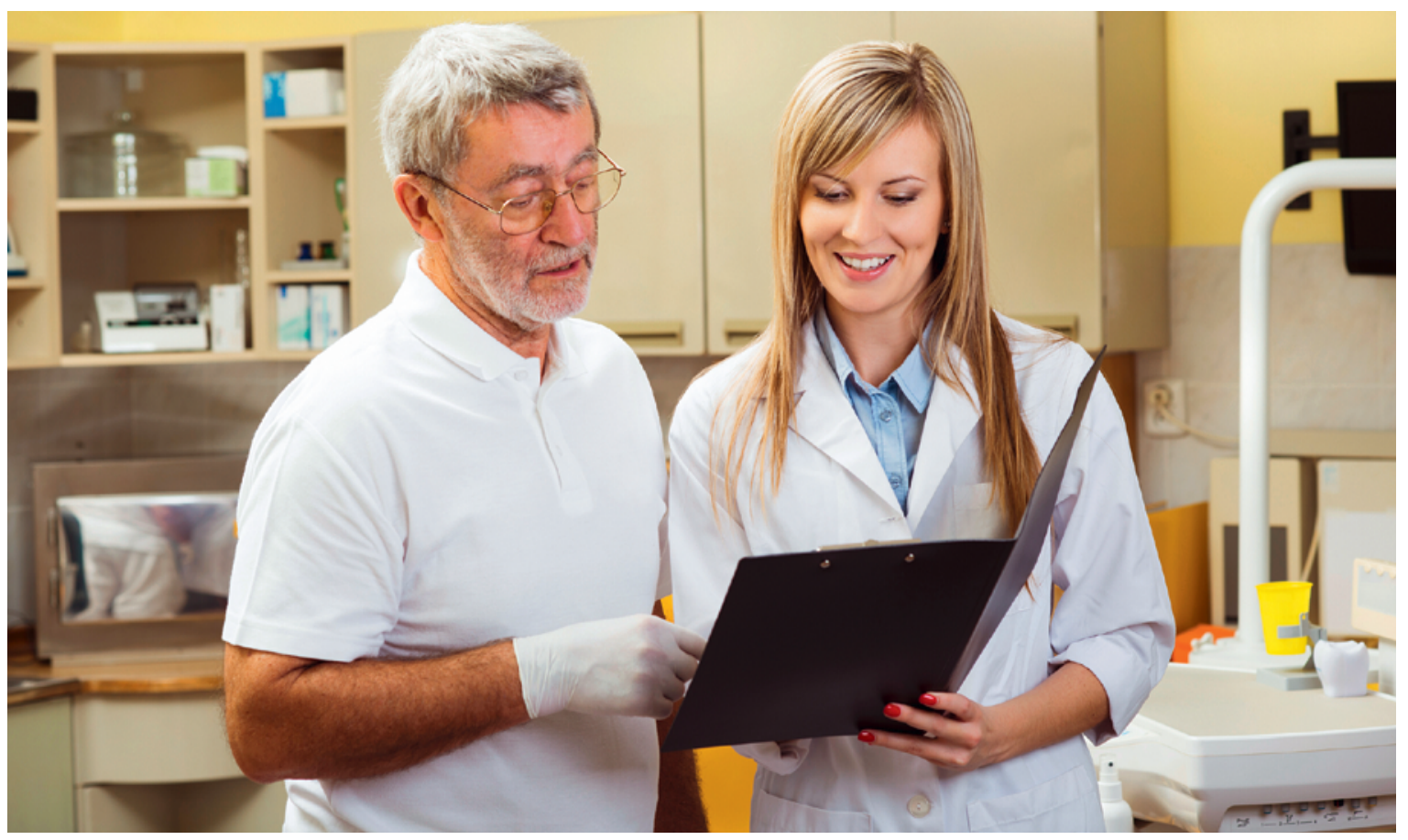

Il y aura toujours moins de médecins de premier recours, particulièrement dans les régions périphériques. Afin de promouvoir la relève auprès des médecins de famille, des programmes correspondants ont été créés dans tous les cantons romands.

Le CRMF estime hautement souhaitable de mettre en place un encadrement de la formation en cabinet et des maîtres de stage par les instituts universitaires de médecine de famille. Ceci permettrait de garantir la qualité et la cohérence de l'enseignement et de la formation fournis.

Le CRMF propose également de renoncer à la limitation du soutien cantonal à six mois par médecin. Ceci permettrait d'augmenter la part de la formation en cabinet, de diminuer l'hospitalo-centrisme et d'augmenter les compétences en médecine ambulatoire.

\section{Les besoins sanitaires de la population et l'évolution de l'organisation du système sani- taire nécessitent d'adapter la formation des médecins de famille.}

Enfin, pour soutenir cette évolution, le CRMF propose que les budgets cantonaux soient réévalués à la hausse afin de faire face aux besoins supplémentaires liés à l'augmentation du temps consacré à la formation en cabinet médical.

Correspondance:

Cursus Romand de Médecine de Famille

Policlinique médicale

universitaire

Rue du Bugnon 44

CH-1011 Lausanne

bernard.giorgis[at]hospvd.ch

françoispilet[at]

vouvry-med.ch l'organisation du système sanitaire nécessitent d'adapter la formation des médecins de famille. Celle-ci doit se dérouler davantage en milieu ambulatoire, notam- ment dans les cabinets médicaux. Dans ce but le CRMF propose d'entamer une réflexion sur la place, l'encadrement et le mode de financement de l'assistanat au cabinet médical. Concrètement, il ne s'agit pas de soutenir au niveau financier un cabinet privé en subventionnant le salaire du médecin-assistant mais bien d'honorer financièrement le travail d'enseignement et de supervision du maître de stage. Ceci est un vrai changement de paradigme.

Enfin, la proposition implique une adaptation des règlements cantonaux d'assistanat et des budgets pour offrir un maximum de places en cabinet. Elle devrait aussi permettre la garantie d'une utilisation qualitative des deniers publics pour la formation en impliquant les instituts universitaires de médecine de famille. Une formation des médecins de famille de qualité induit nécessairement une médecine de premier recours efficiente et de qualité.

Ces changements peuvent servir à augmenter l'intérêt pour la médecine de famille, à améliorer la qualité de la formation postgrade des futurs médecins de famille et, donc, à favoriser leur future installation. Depuis 2007 sur 510 médecins qui ont consulté le CRMF, 330 sont jeunes diplômés. Pour les autres, la moitié est installée six ans après le diplôme de médecin et $98 \% 9$ ans après la fin des études.
Crédit photo

(c) Pojoslaw | Dreamstime.com 